\title{
A Roadmap for Prescribed "Blend- Digit" Personalized Learning - A Collaborated Responsive Approach to Succeeding Generations in the Info Global Age
}

\author{
Ziad Hamdan \\ Hamdan Academy for Higher Education Online, Syria
}

Copyright $\mathrm{O} 2019$ by author, all rights reserved. Author agrees that this article remains permanently open access under the terms of the Creative Commons Attribution License 4.0 International License

\begin{abstract}
Different students have different by nature personality types, cognitive structures and processes, and behavioral modes. Hence, they have no similar aptitudes, needs, purposes, comprehending speeds, academic fields, timelines and locations for learning. As such, to gather "whole" students or large groups in extended large halls or rooms resembling the "Factory Model" is a dire organizational mistake committed against schooling generations since 550 years ago as the German pioneer Guttenberg invented the printing machine 1445. Education systems (ES) instead of employing the new 'discovery' for the welfare of learning generations through giving each student a book, a working manual or handouts to learn individually and in small peer groups as the case of contemporary ICTs, ESs exploited the 'event' for economic benefits. Eventually, they considered the future of generations, which is someday, would be the "future society", a business trade adopting the philosophy and features of the "Factory Model". Thus, the "whole group method" was born and perpetuated in schooling until the beginning of "Third Millennium". Needless to indicate that the 550 years extended of massive learning had caused the loss of millions of the gifted and superiors (who possibly could be future societal leaders and academic pioneers), of the normal (who possibly could be better professionals and skilled leading artisans), and of low achievers (who possibly could be more successful survivals enjoying more dignified living). This Article presents a prescribed ICTs "Blend- Digit" Personalized Learning Approach, which is responsive to individual students needs while adhering to the living demands of Info Global Age.
\end{abstract}

Keywords A Collaborative Approach, Prescribed "Blend-Digit" Learning, Personalized Learning, Succeeding Generations in the Info Global Age

\section{Introduction}

Despite the huge accelerating developments of ICTs since the nineties of the last twentieth century, it is observed that conventional schooling is persisting on some educational misconceptions and obsolete practices. Plato at his Greek Academy 387 B.C. and other schooling leaderships thereafter advocated all students to achieve all subject matters, at specified class periods, according to the same norm referenced criteria and assessment grading levels; as if all school graduates would be science gurus, linguistic theorists, national historians, reforming sociologists, creative engineers, genius mathematicians, or superior medical surgeons!

Time is critical by the beginning of Third Millennium for school systems living the current Global Digital Infocommunication Age to abandon the outdated massive/large group method, which caused huge societal losses throughout education history as a result of student dropouts, failed courses, underachievers, the waste of a bulk of gifted and superiors, more deviants and outlaws, low-quality professionals and mediocre institutional and state leaders (Hamdan (2015 and 1999 in English, and 1988 in Arabic). ICTs have very recently introduced deep changes in the structure and process of schooling. Learner-CenteredParadigm where individual students are the "axis of educational universe", digitizing the curricula, blended, online and cyber clouds approaches of learning and instruction is just example.

SABA 2018 came up with parallel above critiques of conventional schooling by confirming that immense ICTs new products and software, such as (Partly, UNESCO's International Institute for Educational Planning 2018): flipped classroom, connected classroom, Interactive White Boards or Smart Boards, E-readers, smartphones, tablets, digital curricula, tactile and audio technologies for students with special needs, big data, artificial intelligence, internet -of -things, virtual and augmented realities, robotics and 
much more, are impacting the knowledge and skills of schooling. Hence, students and school workers are required to collaborate individually and in teams to fulfill the demands of contemporary teaching and learning.

As such, the requests for technological, social, emotional, and higher cognitive knowledge and skills will rise by 2030 . SABA added, "One thing is certain: the pace of change is intense, and everybody is under pressure to get results quickly". SABA recommended again a direct solution to get out of the educational impasse: "Modernize Learning" by means of contemporary ICTs and the digital transformation of the concept, the factors, processes, and products of schooling.

Prescribed "Blend-Digit" Personalized Learning (PBDPL) is an intact methodology for schooling where the needs, characteristics, and requirements of individual learners determine the goals, tasks, factors, tools, processes, and outcomes of learning and instruction. When optimal commitments of students and faculties' are confirmed in realities, intended achievements and observed results will be likely to have closest statistical values. Hence, quality "Blend- Digit" Learning is realized.

PBDPL is a student-centered approach. Its basic principles are built on self-decided individual students and small peer groups; its psycho-social climate is non-directive where students are inter-independent initiators, collaborators, co-designers, co-decision makers, self-directed performers of learning, and self and co-peers assessors. Teachers and school personnel on another hand play the roles of collaborative providers, co-organizers, coordinators, none-directive counselors, and standby helpers for easing routine problems and reviewers of final achievements.

Further, PBDPL is a multi-track learning process in which individual students could learn the required courses or textbooks according to specific achievement choices as literates in the subject, professional workers, societal leaders, or academic pioneers.

ICTs' accelerating developments and its pending comprehensive impacts are producing more transformations of the factors and processes of schooling. Prescribed "Blend- Digit" Personalized schooling is just one option that is leading to the following assumptions:

- Variations of ICTs are tentatively capable of responding to the Variations of factors, processes, and management needs to be involved in schooling. In fact, as a basic operational principle, "nothing can satisfy variation except variation".

- There will be not much practice in the near future of "whole, massive or large groups learning and teaching" for the sake of individualized and collaborative small peer groups.

- There will be not much practice in the near future of theoretical unified extended written curricula and textbooks for the sake of micro curricular and learning- instructional units and variant schemes of achievement.
- There will be not much practice in the near future of unified daily school schedules, which hold hundreds or thousands of learners in one school area and small classrooms to achieve one synchronous repeatedly study goal/s.

- There will be no compulsory unified curricular content to be learned by all students. Instead, each student will study specific academic content that relates to her/his professional and academic needs for the future and complies with the nature and ability of their special intelligence. One university student protested objectively his professor for teaching him a language-writing course by stating "But wait for professor! I am not an English or journalism major. I don't like to write. I'm studying to become an engineer/biologist/ mathematician/chemist. I won't need to do much writing in my job" (Guessous and Devreugd. 2017, Stevenson, 2017).

- There will be no conventional schooling periods as usual for elementary lower and upper stages, junior and senior highs to which all students should be held regardless of their intellectual abilities and learning speeds. Up to $16 \%$ of students, using PBDPL may finish their K-12 in 6-8 years instead of 15 years. This proposed educational ambition needs of course issuing new regulations on the part of the school district, the ministry of education, national universities and the endorsement of students' families. Needless to indicate that this accelerating alternative necessitates more mentors, school aides, and other management support services. Family and community volunteers, student peers, and part-time recruits could help in succeeding this "shortcut" alternative of schooling.

It is ICTs that enable new ways to cognize, store information, remember, learn, relearn, live, and how to build the personal and professional future. In reality, ICTs are overwhelmingly storming human cognitive ability to comprehend, plan and use the tech adventures (Hamdan 2018); leaving some individuals, teachers, and administrators who are lacking sufficient digital culture, confused, undecided, and waiting for future better or easy to understand and use products.

None-the-less, the main responsibility of above blunders falls in the hands of two parties: first, educators and ICTs specialists for not coordinating/ collaborating efforts to initiate "Blend-Digit" training and reformation of schooling. This in turn could reflect progress upon generations, tech culture, and management of societal institutions including schools and universities.

The second factor of failing or at best slowing down ICTs' reformation of education is governments' management platforms, especially in underdeveloped and developing countries. These administrative infrastructures which are related to education, governance, public order, administration, and political systems are highly corrupted, 
arrogant, negligent, and lacking the sense of direction and responsibility.

Evidently, their daily priorities are non- educational, and the nature and style of daily operations resemble at best the attitudes and practices of "feudal system" (NETO, 2008) dated 476- $1450 \mathrm{AD}$, whose landlords used to confiscate land properties and peoples' labor, to oppress but rarely fair, to treat people as servants and slaves but seldom as citizens or dignified human beings.

The strategic healing solution to counteracting above man-made setbacks is to initiate a "Blend- Digit" personalized schooling (or any promising alternatives) as a reforming overhaul mechanism, which would respond sequentially through two to four generations to students' needs individually and in small peer groups that hopefully could lead to quality ordinary people, leadership, institutions, and more civic-advancing society.

\section{The Concept and Practice of Prescribed "Blend- Digit" Personalized Learning (PBDPL)}

PBDPL (Hamdan, 1999) is an objective well agreed upon pedagogical content for blended learning and teaching. It is like the medical examination: personalized, analytic, prescribed and empirical in content nature, processes, and outcomes. It deals individually with students as they are, understands their ongoing needs and takes them through prescriptions to their desirable achievement ends. These prescriptions utilize different real, blended and digital means that enable students to achieve their learning goals (Hamdan 2018 ${ }^{\mathrm{a}}$ ). It is responsive to students of varied ability, peer groups and as well to the education needs of market place (figure 1).
Further, PBDPL is "a different and more advanced approach of schooling than previous educational practices. It combines simultaneously the merits of prescriptive, blended and online learning tenets and techniques, which embrace when scientifically applied learning free of conventional teachers while moving students to learning choices open to infinity in curriculum options, human resources, tools, techniques, facilities, flexible time and decisions to proceed from one topic to another. The PBDPL gives students open freedom to decide and initiate needed learning individually and in peer groups" (Hamdan, 2018).

Phillips (2014) confirms that different types of instruction require different environment arrangements for learning. As such, several new facilities and equipment of "Blend-Digit" personalized learning should be observed to prove tentatively their proficiency for hosting the PBDPL approach. Further, PBDPL is pedagogically a clinical methodology based on prescriptions, learners' needs, initiatives and self-paced, criterion-referenced learning, self-guided achievement and assessment that guarantee quality outcomes for the majority of individual students.

Zook, 2018 recommends for congruence with contemporary "Blend-Digit" schooling several principles of classroom management and how to be used to teach 'digital natives'. Among these, are:

- 'Start with the easiest principles and get more advanced as goes!

- Establish simple classroom rules.

- Identify common times for classroom disruptions.

- Prevent cheating.

- Get commitments from your students.

- Encourage participation carefully.

- Differentiate your teaching strategies.

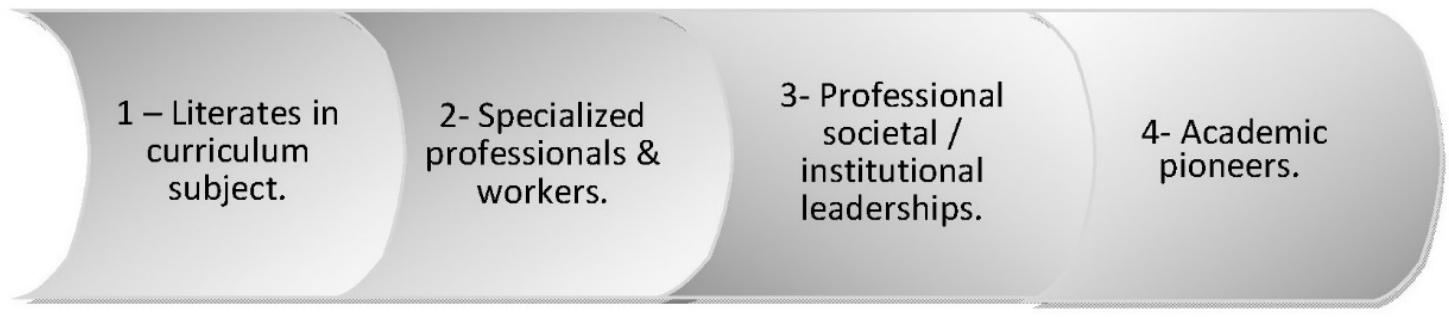

Figure 1. Four Learning Tracks of Personalized Schooling- students as literates, professional specialists, Societal/institutional leaderships and academic pioneers 


\section{Foundations of "Blend-Digit" Personalized Learning (BDPL)}

The underlying educational philosophy of BDPL is a blend of contemporary ICTs student-centered learning paradigm (Hamdan, 2014 and 2018) and the humanistic psychology particularly of Carl Rogers (McDaniel 2018). Hence, the "Blend-Digit" personalized approach is dedicated to the learning welfare of individuals and small peer groups at the time, place, curricular content, pedagogical means and achievement examinations that comply with their declared needs.

In conventional schooling, students learn through mandatory analog curricula and methods, which societal school system wants them to adhere to. Contrary to the blend-digit-humanistic philosophy however, in which learning is ultimately open and individualized.

In congruence with Carl Rogers' philosophy, the "Blend- Digit" Personalized Learning in this article has epitomized his psychological thoughts, principles and techniques. Even before this, Author has suddenly came through Rogers' magnificent psycho-educational heritage such as "encounter groups, personal power, Client-centered therapy, the clinical treatment of the problem child, freedom to learn, On becoming a person, a way of being, empathy, teacher as facilitator of learning but never to dictate it on students, setting a positive climate for learning, clarifying the purposes of learners, organizing and making available learning resources, balancing intellectual and emotional components of learning, teacher's sharing feelings and thoughts with learners but not dominating; student has control over the learning process, student's engagement in learning, learning is based upon confrontations with practical, social, and personal problems, self-evaluation is the principal method of assessing progress or success, learning takes place when the subject matter is relevant to the personal interest of the student, learning proceeds faster when the threat to the self is low, Self-initiated learning is the most lasting and pervasive" (McDaniel 2018).

\section{Prerequisite Factors and Tasks to Initiating (PBDPL)}

Some detrimental factors and tasks for the application of "Prescribed "Blend- Digit" Personalized Learning" in schooling are explained in this paragraph. A committee of ICTs, curricular and assessment specialists, academic resource teachers, and educational psychologists should collaborate in supplying observed shortages, re-designing courses or introducing new assistive technologies, methods and professional knowledge. To guarantee the success of the "Blend- Digit" personalized learning program, PBDPL should be launched only when fulfilling every factor and task.

\subsection{Transforming Computer Analog Instructional Skills into Digital Hard Skills, Then Mastering and Applying Them in "Blend-Digit" Personalized Schooling}

If any school system decides to excel in its ICTs' educational mission, then the first determinant task to consider is to transform the analog paper and hand means of all factors and concepts of conventional schooling into blended and digital form. Next paragraphs give examples in this respect.

These computer hard skills represent the backbone of "Blend -Digit" learning, instruction, management and assessment. Further, the ICTs based concepts and operations of equipment and facilities such as augmented and virtual realities, connected classrooms, study carrels, video conferencing, cyber clouds, blended and online peer projects and discussions, and digital curricula are of high priority.

Hard skills are specific knowledge and abilities that are achieved through blended education and training. In "blend-digit" schooling, the following skills are highly recommended for teachers, students and personnel to achieve as literates at the least degree possible (KERR.2017).

\subsubsection{Computer Technologies}

Important technology-related hard skills examples are basic to professional "Blend- Digit" teachers, ICTs specialists and technicians, which are:

- Microsoft Office Suite.

- Social media.

- HTML.

- Analytics.

- Key tables.

\subsubsection{Hard Communication Skills}

Examples of hard communication skills that are necessary for "Blend- Digit" schooling personnel are:

- $\quad$ Foreign language.

- Digital communication.

- Copywriting.

\subsubsection{Data Analysis}

The abilities to analyze data and then use information for the benefit of "Blend- Digit" schooling personnel are:

- Data mining.

- Data presentation.

- Resource management.

- Data engineering.

- Database computer programming.

\subsubsection{Certifications and Licenses}

Some common types of certifications and licenses 
include:

- Learning management systems, such as Blackboard, Canvas, or Eagle.

- Data management systems, such as Colleague

- Healthcare related licenses.

- Certificates, awards, badges, and industry-recognized credentials.

\subsubsection{Marketing}

A number of hard marketing skills that have become incredibly sought in recent years are:

- $\quad$ Search Engine Optimization (SEO).

- $\quad$ Search Engine Marketing (SEM).

- Marketing Campaign Management.

- Google Analytics.

- Content Management Systems (CMS), such as Word Press.

\subsubsection{Project Management}

Some important hard project management skills include:

- $\quad$ Agile Methodologies, such as Scrum.

- $\quad$ Project Management Software, such as Trello and Zoho.

\subsubsection{Design}

Here are some examples of some of the hard design skills that are most desired by employers:

- User Interface (UI) Design.

- User Experience (UX) Design.

- $\quad$ Adobe Creative Suite (Photoshop, InDesign, etc.).

- Digital Product Design Software, such as InVision and Zeppelin.

\subsubsection{Cloud Computing}

Some examples of hard skills that employers look for when hiring cloud-computing professionals are:

- Cloud Architecture.

- $\quad$ Storage and Data Management.

- Networking Communication.

- Cloud Middleware Technologies.

- Cloud Applications, such as JSON, Rest, and RPC.

\subsubsection{Mobile \& Web Development}

Some examples of the kind of hard skills that you need to become a successful developer:

- Software Revision Control Systems.

- Android Development.

- $\quad$ iOS App Development.

- Web Architecture and Development Framework.

- Angular and Node Apps.

\subsubsection{Network Structure \& Security}

For schooling network security, IT professionals must have the skills to protect the educational, academic and digital data under their responsibilities. Examples of hard skills that should be achieved here are:
- Encryption Algorithms

- Authentication Systems.

- $\quad$ Risk Assessment.

- Cryptography.

- Virtual and Host-based Firewalls.

\subsection{Counteracting Operational Challenges to (PBDPL)}

These operational challenges could be summed up in the following six points:

- About $50 \%$ of the top U.S. schools require a course in technical communication.

- Budgetary constraints.

- Credit hour constraints.

- Lack of faculty expertise in teaching technical writing, even more so for graduate teaching assistants.

- Time constraints for students and faculty.

- Faculty motivation. e.g. saying "not our job" (Guessous and Devreugd 2017)

\subsection{Counteracting Student Academic Shortcomings in (PBDPL)}

- $\quad$ Focus on minor details and fail to see the big picture or main results.

- $\quad$ Focus on facts and details but lack analysis.

- $\quad$ Reach conclusions without directly referencing their data or analysis.

- $\quad$ Issues with organizing and presenting information in a logical manner.

- $\quad$ Generating good quality tables, graphs, and figures.

- Writing abstracts!

- Writing mechanics problems (spelling, grammar, punctuation); citing references

- $\quad$ Long, convoluted sentences.

- Team dynamics.

- $\quad$ Nonnative speakers (Guessous and Devreugd 2017).

\subsection{Counteracting Barriers to E-learning Practice in the Middle East}

Mirza and Abdulrahman 2011 summarized the barriers to e-learning in the Middle East in the following:

- Low digital culture among different sects of Arab societies. Few people have knowledge of e-learning systems, including those at the academic level and employees at governmental and private organizations.

- Low Internet penetration rate by the public, which is attributed to unsystemic high initial costs associated with Internet access, low speed and quality Internet connections,

- $\quad$ The fear that Internet connections would bring-about immoral values and corruptive conducts to the family.

- $\quad$ The warning calls from conservative religious clerics who continuously propagandizing against the dangers of the Internet will bring to the society. Many adhered to the warning 
- Low public esteem for online learning. The online Models of e-learning adopted in the Middle East 87 degrees generated negative reactions against online graduates appeared in less job opportunities comparable to traditional degrees.

- The learner's attitude and lack of prior knowledge of IT use are major factors that affect students' acceptance of e-learning.

- The lack of online repositories of educational materials in the Arabic language. A great percentage of faculty members may not be capable of creating such materials; hence, courses would not lend themselves to eLearning.

- The desperate need for training workshops to bridge the technology gap and improve faculty self-perception, professional knowledge and skills.

\subsection{Forming and Reinforcing "Blend-Digit" Learning Policies}

Many institutions including schools base their activities on the expectations of what an "average" person is and assume that each individual person is similar to the average. In fact, this assumption is often misleading. In psychology, there is no such approved valid and reliable concept called "average" in schooling (Hamdan, 2018 ${ }^{\mathrm{a}}$; Rose, 2016).

For example, students, who get $\mathrm{B}$ grade while are differing in several individual characteristics, life conditions, and family backgrounds such as socioeconomic status, culture, aptitude, health condition, personal ambitions and hobbies, can't be treated in lieu of the "average" student or person since the content, the brain and physical internal processes and implications for the future are non-identical among B students. This creed of "end of average" is exactly similar to the belief and principles, which "Blend-Digit" methodology advocates and operates in schooling accordingly.

Moreover, "Blend-Digit" Learning Policies are based generally on the following operational principles (Hamdan, 2018 ${ }^{\text {a }}$ :

- 'eLearning is open to infinity: "Blend-Digit" approach combines face-to-face and online means for the service of learning and instruction. Hence, individual students and peer groups have total flexibility to determine and plan what they tend to learn at the time and place, which are convenient to them whether being inside or outside their schools or elsewhere.

- Multi learning tracks are the central philosophy of "Blend-Digit" methodology that responds through "personalized learning" to student individual differences in aptitude, attitude and personal academic/ professional choices for the future. The learning options available to students are of four options: literates, professional specialists, societal / institutional leaderships and academic pioneers.
- Multi-stage schooling: it covers all major educational phases of schooling, which generations are psychologically and physically ready to build their intellectual and professional skills, and to earn ambitious certifications. These stages encompass specifically nursery and kindergarten, lower elementary, upper elementary, junior high (middle school), senior high (secondary school), BSc, MSc/ MA, and Ph.D. studies.

What distinguishes the current research topic as is indicated above is the spiral continuity design of its content in that each schooling stage from nursery up to the Ph.D studies has a continuum interrelationships. Each consecutive stage offers students goals, knowledge, values, skills, learning, and achievements that are highly and inductively related.

This author believes that a cardinal reason beyond the failure of education systems stems from their negligence of promoting the tenets of inter-dependence and continuity between schooling curricula or courses and learning-teaching strategies used in consecutive grades and stages. Needless to emphasize that these curricular and pedagogic principles provide schooling with meaningful preconditions of applicability, validity, and reliability necessary for effective students' achievements.

\subsection{Building Professional "Blend-Digit" Competent Faculty}

ICTs Global Age is, in reality, the "period of human diversity and connectivity". For this, individuals and groups have the ability and digital means to connect, interact and learn from each other regardless of race, culture, geography, and topic. These online communicational flexibility merits while easing the exchange of ideas and mutual psychological proximities, require from groups, especially in connected classrooms to interact openly, to tolerate differences among each other and accept themselves, as they are individuals and groups if they seek effective "Blend-Digit" learning and teaching to occur.

The widespread practice of ICTs in education and the current "Blend-Digit" learning approach have made it possible to revoke the roles of "corporal and large groups' teachers". Their participation in the "Blend-Digit" schooling should be conditional with updating their ICTs professional knowledge and skills'. UNESCO's IIEPInternational Institute for Educational Planning (2018) confirmed, "ICTs can impact student learning when teachers are digitally literate and understand how to integrate it into curriculum".

In educationally advanced countries, students use usually diverse ICT tools in daily communication, life activities and schooling. For teachers who are digitally literate and trained to use ICTs, can lead students to higher order thinking skills, provide creative and individualized 
options for them to express their understandings, and be better prepared to utilize technological changes in schools, society and the workplace (UNESCO's IIEP 2018).

\subsection{Building Intercultural Competent Faculty}

Samdperil and Gunther 2017 defined intercultural competence as "the ability to relate and communicate effectively when individuals involved in the interaction do not share the same culture, ethnicity, language, or other common experiences." Teachers and students diversify intercommunication based on 14 dimensions according to Samdperil and Gunther: race, ethnicity, gender, age, religion, socio-economic class, ability, sexual orientation, language, appearance, geographic locations, education, learning style, and work profession.

Wikipedia 2018 added that 'Cultural competence is the ability to interact effectively with others of different cultures. It consists of four components:

- Awareness of one's own cultural worldview,

- Attitude towards cultural differences,

- Knowledge of different cultural practices and worldviews, and

- Cross-cultural interactive skills'.

"Carl Rogers was a gifted teacher" with a merited approach of one-to-one professional encounters. He viewed himself as a facilitator who creates the environment for engagement, emphasizing throughout positive attitudes, and considering explorations and encounters more significant than the methods that are employed.

Carl Rogers on the interpersonal relationship through the facilitation of learning was merited as a real person facilitator who applied to prize, acceptance, and trust. It is as prizing the learners, prizing their feelings, opinions, and persons. Rogers was caring for the learners as dignified persons, having worth in their own rights regardless of their personal differences and cultural backgrounds. (Smith, 2004)

Irish and Scrubb 2012 and Lynch 2018 summed the importance of sensitive and responsive instructors for comprehending the differences among individual students. They need to strengthen specific competencies to acquire and teach all students, such as the following seven professional behaviors:

- Investigate their own culture and reflect upon students cultures.

- Enable critical reflection by students.

- Demands respect for others.

- Learn about individual students.

- Use of intercultural communication skills. Culturally competent instructors are active listeners and acute observers

- Investigate how cultural differences might affect academic performance.

- Use of focused activities and intentionally structured environments including an understanding of norms, values, and traditions that have informed other's worldview and learning behaviors.

\subsection{Development of Blended and Digital Curricula}

Curricula are the primary factor besides teachers and students to determine the concept and operation of schooling. It simply conveys the academic content of teaching and learning. In the current Info Digital Age, transforming analog curricular documents into digital and blended formats is of ultimate necessity.

"Blend-Digit" curricula in forms of blended learning documents, online courses, electronic textbooks, and online programs are replacing traditional paper textbooks. In addition, digital curricula when aided by digital tools and resources (Clint 2018) such as videos or movies, word processing documents, slide presentation software, electronic reference materials, whiteboards, tablets, and cellophane apps could be highly appropriate and responsive in delivering instruction and in maintaining several classroom purposes.

Digital curricula as becoming transnational and global learning- teaching devices used by different cultural school systems across the World, should take into account through redesigning the digital shifts from analog to blended and online, the following principles (Samdperil and Gunther 2017):

- "Increase awareness of racial and ethnic variations in education and the importance of sociocultural factors on beliefs and behaviors.

- Identify the impacts of race, ethnicity, culture, and class on the decision-making of learning and instruction.

- Develop skills for cross-cultural assessment, communication, and negotiation."

- $\quad$ Further, several additional tasks should be considered for reviewing, renewal and modification of curricula to fit the nature and demands of "Blend-Digit" schooling. These are (Samdperil and Gunther. op. cited):

- 'Countering the Backward Attitudes against "Blend-Digit" Learning.

- $\quad$ Preparing "Blend-Digit" Human Resources.

- Preparing Professional Technical services for "Blend-Digit" Learning.

- $\quad$ Preparing "Blend-Digit" Learning Support Services.

- $\quad$ Preparing "Blend-Digit" Environments- Facilities and Equipment to host learning.

- Providing teachers and students access to "Blend-Digit" learning resources such as school and public libraries, ICTs centers, and transnational video conferencing and discussions.

- Mainstreaming samples of "Blend-Digit" prescribed /personalized learning by means of 'hot-house trials', individual schools field experimentation initiatives, and transnational school applications. 


\section{A Roadmap for Implementing Prescribed "Blend-Digit" Personalized Learning (PBDPL)}

The Roadmap of PBDPL includes eight systemic steps; appear as follows (Hamdan 1999):

\subsection{Student Visitation to the Academic Counseling Center (ACC) to Supply Background Data and Consult with the Teacher}

This step encompasses the foundation of three steps of prescriptive "Blend-Digit" personalized schooling. For students who are already part of the school system, two types of visits are possible for them: the first is a short visit, which will be an automatic easy routine, taking only a few minutes to accomplish.

Visiting the ACC should be straightforward, equitable and organized. To comply with these criteria means that all students, young and adult, regardless of their personal prejudices must have easy access to the ACC according to some objective principles. A widely used one is "first comes, first served."

However, appointments to visit the ACC preferably can be made by any direct or online means. Full-time secretary and support staffs should be available to receive distant phone inquiries or in-person requests. Regular records of names and visiting purposes, allocated time, and date appropriate for each case, are necessary to keep concise records of daily visits.

It is highly recommended in this context that articulated blended records should be maintained, assigned to individual students and it states clearly: student's name, grade level, or status, the mission or the learning goals, allocated time and date for the visit, the date of previous accomplished topics, tests to be administered, specific questions or concerns to be addressed, study materials and tools to be used, and any other data that may benefit student's visit, the counseling process and the development of prescriptions as a whole.

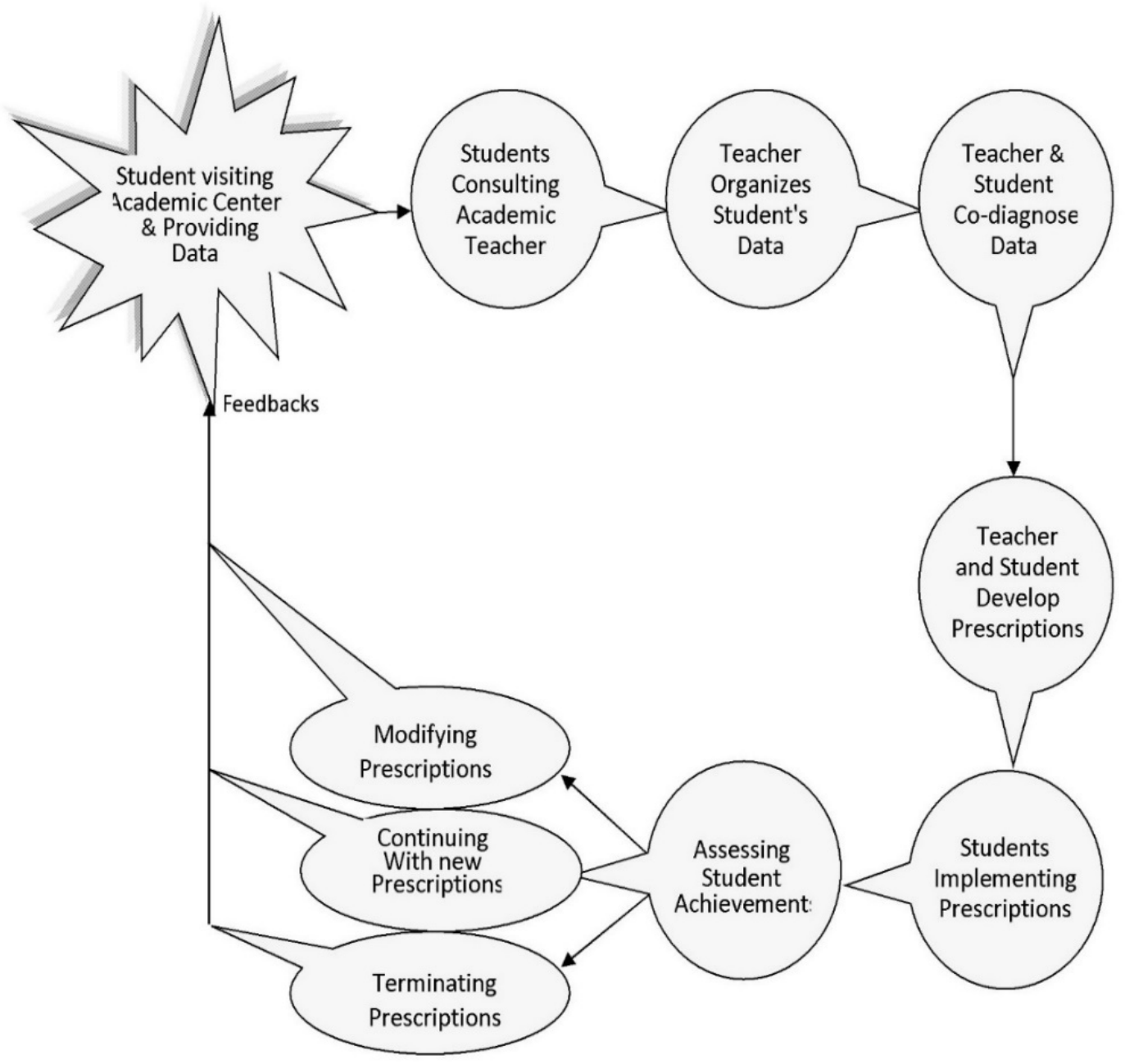

Figure 2. ICTs Integrated "Blend-Digit" Personalized Learning 


\subsection{Development of Prescriptions for Personalized "Blend-Digit" Learning}

Joint consultations and counseling of academic teacher and individual/ peer group students will pave the way to developing "Blend-Digit" prescribed learning. One basic type of learning that prescriptions should emphasize is promoting the values and skills of critical thinking (Nilsson 2018), in contrast of the conventional approach where lecturing, reciting, repetition are wasting any creative mind.

Contemporary educators strongly recommend critical thinking be central to students acquiring critical thinking skills. We must ask students challenging, open-ended questions that demand genuine inquiry, analysis, or assessment critical questions.

Moreover, discovering students' answers to social and emotional questions can help to create positive conditions for learning. Know about students because knowing them will greatly influence teaching. They reflect the conditions necessary for students to learn, be happy, feel relevant, and be flexible (Elias2018).

Blended prescriptions, in order to be psychologically and operationally viable, several criteria must be noticed when being prepared electronically for students, such as (Hamdan, 1999):

1. Writing the prescriptions in clear sound language, readable by students and school personnel alike. Just as the medical prescription, the content of the "blend-digit" prescription must be directly understood with no need for further interpretation or clarification.

2. Writing the prescription in operational form, behavioral blocks, formative steps and/or the performing logical sequence are essential features for the organization of prescriptions' content.

3. Each implementation segment of the prescription should be simultaneously self-fulfilling and independent. While the segment is implemented to serve specified psycho-educational purpose(s), its content and achieved results interweave at the same time with other prescription segments, which together form a logical chain within the total "blend-digit" schooling sequence.

4. Writing the prescription in a concise length that is appropriate to its nature and content, implementation requirements and comprehension-perseverance abilities of students. Academic prescriptions, in general, tend to be relatively easy to understand, straight forward to perform, and consequently may appear moderate in length. Psychosocial and behavioral prescriptions, on the other hand, are complicated in nature and factors; therefore, they must be carefully detailed to avoid any misinterpretation of contents on the part of students and support workers alike.

Cognitive styles interfere also in the length of prescriptions. Students who for example learn by sequential details or who have lower intelligence or special aptitudes than normal peers are in need of prescriptions that are detailed in content to satisfy their cognition. Bright and highly independent students, on another hand, can work with little details, e.g. brief performance guidelines or instructions will suffice to achieve the psycho-educational outcomes.

5. Writing the prescription in a mutually exclusive form. This means that the prescription per se and each learning block within its content should embody all factors, processes, and means which could be used to realize the stated goals.

\subsection{Employing Primary Methods Necessary for Personalized "Blend- Digit" Learning}

Ten primary methods could be used:

\subsubsection{The Prescribed Educational Method (PEM)}

This method (PEM) is the basis for operating "blend-digit" schooling approach from the beginning to the end. Without it, blend-digit schooling will convert to a boring didactic task, fruitless educational theorizing or to a dead human endeavor.

The PEM combines the principles and procedures of the clinical practice of medicine and psychology. It is a descriptive road map for goals to be achieved, the materials to be used, activities and assignments to be undertaken, counseling and guidance services to be consulted, and learning assessment techniques available to use and pursue leaning.

\subsubsection{Blended Active Learning Classroom (BALC)}

The integrated use of connected technology is enabling blended approaches to teaching and learning. The physical space, the digital space, the Blended space can change for better what is possible in the classroom. It resembles generally the standard "Connected Classroom"(CC).

$\mathrm{CC}$ includes technologies such as internet lines, PCs, data projector and visualizer. It has available wireless internet so that staff and students can utilize their devices in schooling. However, it does not typically contain specialized equipment so that CCs enable teachers to work more closely with their students in seminars, discussions, brainstorming and workshop modes, as examples (Middleton and Glover 2017).

\subsubsection{White Board Learning (WBL)}

With digital whiteboards, flexible active classrooms are developed to include digital screens. Depending on their physical size and shape, multiple 'repeater' screens or projectors are being included to ensure students visualizing what is being presented regarding the directions are facing.

Whiteboards are keys to the active classroom. They offer extensive space within the room for students to work 
creatively to construct visual representations of their learning e.g. mind maps, lists, and process diagrams. However, technicians should consider moving furniture away from the walls to support whiteboard usage and encourage small group activities during teaching sessions without causing undue disruptions (Middleton and Glover 2017).

\subsubsection{The Personalized Tutorial Method (PTM)}

Tutoring lies in the very heart of prescribed blended schooling approach, since the latter is in itself a diagnostic individualistic methodology of education, relies heavily on face-to-face communication and utilizes special needs and interests of individual students. The personal tutorial method (PTM) by which academic and behavioral specialists tutor individual and small groups of students according to their declared needs, is considered a very basic tool for operating blend-digit schooling.

\subsubsection{The Guided Independent Study (GIS)}

Right after the student gets an individual prescription; he/she may find in need of some tutoring in a specific topic or to an instructional advice, knowledge or experience.

When finishing tutorial sessions, the student is free to decide to accomplish what he desires of the required tasks in her/his prescription, and to employ from working alternatives, the human, and material services which are compatible with their available daily time, their cognitive styles, the nature of the psycho-educational tasks and finally with personal preferences.

Throughout the independent process of achievement, individual students may need however sporadic guidance. The result forms the elementary concept and practice of the guided independent study (GIS).

\subsubsection{The Small groups' Method (SGM)}

Prescribed blended schooling is not an isolated human activity; it is rather an interactive face-to-face and students' active participation approach to education. Individual students according to their own needs and initiatives interact with each other and with school personnel directly and digitally to achieve specific psycho-educational ends.

Where large groups' methods have almost no role in blend-digit schooling, small groups study (SGM) comes next to guided independent work. Peers who share similar attitudes, backgrounds and/or psycho-educational needs tend to form study groups of their own. Two to four or five students gather themselves together in one cooperative educational clique or league. The group when carefully and indirectly supervised will provide clinical blended schooling with a highly powerful vehicle for social, behavioral and academic developments of students.

\subsubsection{The Online Method (OLM)}

The online method (OLM) is the seventh principal method discussed in this paper. It is probably the most sophisticated mechanism used today for the implementation of digital and blended schooling. When the student or the faculty uses an electronic device, e.g. mobile, iPad, tablet, internet line or Wi-Fi, laptop, or any other digital tool, they are using the online method. Several working points related to this method are explored in the next paragraphs.

\subsubsection{Smartphones and Tablets Exert a Profound Impact on the Work and Lives of Most People}

In the U.S, individuals check on their mobiles about 150 times a day and it is estimated that that 90 percent of full-time employees use mobile devices for work. SABA 2018 noted, "by 2019, 69\% of the people in the world will be mobile device users". This indicates tentatively how significant the role of mobile devices such smartphones, tablets, palm machines and laptops is in the process of learning and achievement of "mobile learning" or "m-learning" (LaMotte 2018).

Actually, these e-learning hand-tools have paved the way for the rise and application of a new e-method that is "bring your own device (BOYD)". As such e-education alternatives through BOYD have eased and diversified media, time and locations for students' learning to unprecedented degrees.

\subsubsection{The Mobile Method (MM)}

Global mobile traffic will be three times faster than desktop in the period of 2014-2019 and 50\% of digital phone applications for learning (Docebo. 2018).

- Heavy use of BOYD (bring your own device). This mix of digital devices provide mobile learning with benefits of:

- Flexible work environment.

- More learning.

- Fewer costs.

- Intensive use in learning content delivery.

- More companies use mobile to deliver learning.

- Use for video learning which will reach $80 \%$ of the World's internet traffic.

- More study content is prepared for mobile learners.

- Intensive use of gamification in mobile learning. Gamification gives learners control, flexibility and convenience to learn anywhere, anytime and generate more exploration, decision-making activity, and learner-centered experiences.

- Personalize the process of learning. Every learner, young or adult can by a key touch start the learning path of her/his choice.

- Encourage learners to participate in social and collaborate efforts meaningful to learning contexts.

- Allow learning offline to become online later. This could happen when power or the Internet or both are off as the case of many Developing countries and rural areas, students could turn to a school, a college, education ministry, or private business platforms that 
allow them to learn offline and then ensure their achievements automatically online without losing any bit of what has been learned.

- Eliminate most administrative barriers of learning, relearning and upgrading learning.

- Individual students have the right to choose whatever they desire in coordination with a nondirective teacher and /or a learning counselor.

\subsubsection{The Field Study Method (FSM)}

Community resources and local environments are probably the richest media for learning and instruction. The experiences of these resources provide concrete, real and familiar to students, far more than any other oral, written and technological encounters which students face unwillingly sometimes in school settings. The context of real and blended schooling, however, is made up of both community and school environments, allowing playing a major non-traditional role in the course of education. The (FSM) is generally looked upon an effective tool for deep learning and instruction.

\subsection{Implementation of "Blend-digit" Prescriptions for Personalized Learning}

Partridge and Ramaswamy 2018 proposed ten strategies for implementation of new e-Learning program, appear in the following:

- Specifying expectations for the e-learning program.

- Validating compatibility across education solutions.

- Building the right team.

- Content selection and organization.

- Creating a realistic, detailed timeline.

- $\quad$ Prioritizing requirements \& expectations.

- Pre-launch testing.

- $\quad$ Training \& ongoing support.

- Marketing your success.

- Establishing a learning culture.

Michael and Others 2011 identified 14 conditions that are decisive for technology in learning. They are summarized in the following points:

- $\quad$ Shared vision.

- Empowered leaders.

- Implementation planning.

- Adequate and consistent funding.

- Equitable access.

- $\quad$ Skilled personnel.

- $\quad$ Ongoing professional learning.

- Technical support.

- Contemporary curriculum framework.

- Student-centered learning.

- Systemic assessment and evaluation.

- $\quad$ Engaged communities.

- Support policies.

- $\quad$ Supportive external context.

\subsection{Implementation of "Blend-Digit" Prescriptions by 3 Minute E-learning Technique}

This is an alternative technique (Jimenez 2006) that enables an immediate action for rapid development and delivery of "Blend-digit" prescriptions and contributes to quick skills acquisition.

When adopting 3 Minute e-Learning, Jimenez recommends 15 guiding principles:

- $\quad$ 'Concise

- In-chunks

- Rabid

- Quick- to learn

- Effective

- Low cost'

- Quick access

- Instant use

- No memorization

- Random learning

- Optional, as needed

- Control of learning by learners

- Organizing content for e-Learning to enable the learner to access the knowledge and information that help finding application or performance ideas.

- Apply them quickly and rapidly.

- Make sure that content is short, making them available, reducing the cost of development and increasing the speed of production.

However, each student is expected throughout the implementation to apply the instructions and content of the prescription to meet the needs for which he/she has sought through the Academic Counseling Center. The student does that like the physical health patient who takes the prescribed medicines one after another according to specified instructions until getting the required health results.

After brief counseling with the academic teacher or other school personnel concerning the best ways to accomplish the tasks stated in the prescription, the student leaves the academic resource center to start the actual process of new learning, or the needed behavioral, social and personal improvements.

\subsection{Assessment of Students' Achievement of Personalized "Blend-Digit" Learning Prescription(s)}

The assessment methodology of "Blend-digit" learning is a collaborative criterion-referenced measure. Students themselves, peers and teachers employ the needs/ achievement objectives agreed-upon in the inputs stage as absolute criteria to which formative and summative achievements are compared, evaluated, and produced decisions to move successful students to another level or new course, or introducing improvement revisions on learning program, environments, methods, and services for 
better final results.

In "blend-digit "schooling, individual students and small cooperative peer groups carry out the responsibilities of assessment tasks of learning achievements. Large group methods, on the other hand, are rarely practiced. Techniques such as individual self-initiation, peer review, analytic research, collaborative, formative and summative examinations, rubrics, criterion-referenced and classroom assessment techniques are generally employed.

The counselor teacher assumes however, a final external surveying checkpoint using whenever is necessary quick face-to-face, blended and online assessments to decide for each student one of the following (Hamdan 2016):

- Achievement is successful. Hence, the student is qualified to move to learn another task.

- Achievement is inadequate, and improvement is necessary, or

- Achievement is flunked. Hence, the student is obliged to repeat learning the task.

\subsection{Meta-evaluation of Inputs, Processes, and Outputs of "Blend- Digit" Personalized Learning}

This task is, in essence, the evaluation of previous assessment or the evaluation of previous evaluation for validating the factors, processes, and outcomes of personalized "Blend- Digit" learning. It involved three minor undertakings:

- Re-evaluation of inputs, processes, and outputs of "Blend- Digit Personalized Learning".

- Re-evaluation of quality of students' learning against specific successful performance criteria.

- Re-evaluation of learning consequences of liberating local learning to open transnational choices.

Learning assessment, especially the "formative" has a significant role in establishing student achievement. However, when this building assignment is usually delegated to students individually and in small collaborative peer, it becomes essential to train learners for mastering self and interpersonal professional assessment tasks throughout the formal and informal processes of schooling.

While these formative endeavors accumulate evidence for mastering the studied content and options for improving learning, it empowers students as self-regulated and life-long learners. Box 2018 proposes for students to build self and peers collaborative assessment skills, strategies such 'anonymous examples of strong and weak learning performances' by means of rubrics, real samples of learning and analytic critical discussions, and "TSAR Think, Share, Advise, Revise".

\section{Epilogue}

Formal education of k-12 schooling, colleges and universities has persisted in its analog, didactic, and large group methodology for thousands of years since Plato's Greek academy. However, several developments and inventions have emerged at the educational scene throughout the above-extended era of World education.

Examples of these inventions (Kryss Tal 2018) are: Paper, Modern Numbers and Zero, Guttenberg printing machine, Postal Service, Newspaper, Calculus, Typewriter, Television, Videophone, Mobile Phone, CD, Video Tape/Recorder, Satellite, Internet, ICTs, E-Mail, Personal Computer, laptop, tablet, BYOD, wireless communication, digital, blended and cloud methods, Nano technology, and public issues of democracy, justice and human rights.

Despite of above examples of inventions, schooling remained outdated business, conventional and irresponsive to many digital and blended emerging alternatives. Education in general is the most conservative profession in regard of renewals and reformation tendencies compared to businesses of communication, industries, private trades, and administrative agencies.

Urgent integration efforts of ICTs in schooling should be launched to produce deep up-to-date changes in the inputs, processes and outcomes so that k-12 and higher educational institutions keep its constructive roles in progressing generations, communities and national corporations.

However, what seems paradoxical concerning the low quality of conventional schooling is the limited utilization of digital technologies. While ICTs are abundantly available in both hardware and software, fruitful efforts to utilize digital products for reforming schooling are rarely observed. Hamdan $\left(2018^{\mathrm{b}}\right)$ confirmed that despite "excess ICTs production and availability for education, limited organized integration attempts of ICTs in schooling were apparently successful, no serious educational reforms were evident, and no venture to replace Teacher-CenteredParadigm with Learner -Centered Blend- Digit Approach has been materialized".

Other additional problems hindering the quality dissemination and implanting ICTs in the structure and operation of schooling are:

1. The miscommunication and the lack of coordination among different parties related to digital technologies.

For example, the enterprise who invents, who develops, who trades, who adopts for students and teachers, who assesses the impacts on achievement, and who mentors and maintains the field services have no or limited personal or professional knowledge of others before. Evidently, they function independently or in isolation from each other, contrary to their individual shared welfares (the progress of generations, institutions and local communities) which call for devoted collaboration through maintaining the quality of ICTs oriented schooling.

However, while the nature and strict confidentiality needed for private industries and the art of inventions may justify the "solitude work"; Nevertheless, as products 
transform to public domain, then the parties involved should embrace open interaction, info exchange and collaboration to comprehend the realities of required ICTs schooling missions, and the potential role(s) expected from each to advance schooling. One educational fact could be remembered is that "if teachers and students are seated beside each other in the classroom for a full year without interaction, info exchange and collaboration, schooling will never occur. This conclusion applies to all parties involved in ICTs integration in schooling.

2. Missing strategic tools for initiating and maintaining sustainable collaboration for ICTs' based education.

What is urgently needed for overcoming the stalemate of active collaboration and interaction of all ICTs parties involved at local, transactional and global levels, are three procedures: ICTs platforms, school digital societies (SDSs) and "hot trials school laboratories". These three approaching mechanisms reflect positive educational, social and professional implications on schooling.

ICTs platforms are recommended to utilize quality retired and volunteered professors such as educators, ICTs specialists, academic majors, educational psychologists, sociologists, statisticians and others. The main duties of these faculties are to plan and develop the platform programs, lead discussion meetings at local, transnational and global levels, and collaborate with colleagues to reach effective "blend-digit" decisions for ICTs successful integration in schooling.

On another hand, SDSs are organizational and operational affiliates of schooling affairs locally and transnationally including the advancement of "studentship" and "blend-digit" learning and instruction. Four types of SDSs are involved: local, national, continental and Global. These SDSs are gradually inter-related according to membership sizes and responsibilities, thus two additional types merge: DSSs leagues and congresses (Hamdan, $2018^{\mathrm{b}}$ ).

Finally, the "hot trials laboratories", which support school facilities and equipment that provide instant experimentation of ICTs integration and tentative examination of the validity and impact of "blend-digit" knowledge, tools, techniques, methods and other techno-educational alternatives in semi-normal school settings.

The establishment of these new facilities depends on financial capability of the school. Financially stable school could have its own laboratory, while others of less economic capacity, availability of wise leaderships could enable several schools to fall in close geographic proximity to form a collaborative trial laboratory and fulfill their quick laboratory experimentation needs.

\section{REFERENCES}

[1] Box, Cathy. 2018. Assessment for Learning: It Just Makes
Sense. The Teaching Professor, Faculty Focus, Magna Publications. https://www.facultyfocus.com/articles/educat ional-assessment/

[2] Clint, Frank (Instructor).2018. Digital Curricula vs. Digital Tools: Definition \& Uses Chapter 19 / Lesson 4. Study.com. https://study.com/academy/lesson/digital-curricula-vs-digit al-tools-definition-uses.html

[3] Docebo. 2018. 10 Mobile Learning Trends You Need to Know Before 2019. Docebo-WP-10 Mobile Trends-ENG-08(1).pdf

[4] Elias, Maurice. 2018. Important Questions to Ask Your Students. Edutopia. https://www.edutopia.org/ https://www.edutopia.org/article/important-questions-askyour-students.

[5] Guessous, Laila and Devreugd, Leanne. 2017. Best Practices in Teaching Written and Oral Communication Skills to STEM Students. http://www.csss-science.org/teac h.shtml

[6] Hamdan, M. Ziad. 1988. CLINICAL EDUCATIONToward a Successful Tool to Superiority \& Overcoming Achievement Deficiencies. Damascus: Modern Education House (in Arabic).

[7] Hamdan, M. Ziad. 1999. Re-schooling Society in the Digital Age - with a Clinical Personalized Approach. Damascus: Modern Education House.

[8] Hamdan, M. Z. 2014. A Paradigm of Transnational Collaborative neo-Blended Learning: toward an exchange theory of growth need - responsive source transactions. Paper presented to the 13th European Conference on e-Learning ECEL-2014 held at Aalborg University, Copenhagen, Denmark at 30-31 October.

[9] Hamdan, M. Z. 2015. Blended Achievement in Transnational Schools as Collaborative

[10] Learning Communities- Toward a Systemic Assessment Framework. International Journal of Modern Education Research. Vol. 2, No. 6, pp. 88-97.

[11] Hamdan, M. Z. 2018 . A Non-Failing "NeoBlend-Digit" Schooling Free of Conventional Teachers: A Blueprint for a Futuristic Learning Open to Infinity. International Journal of Contemporary Education, [S.1.], v. 1, n. 1, p. 64-86, Apr. 2018. ISSN 2575-3185. Available at: http://redfame.com/j ournal/index.php/ijce/article/view/3246/3417>.

[12] Hamdan, M.Z. 2018 . ICTS INTEGRATION PLAN FOR LIBERATING LOCAL MASSIVE LEARNING INTO DIFFERENTIATING STUDENT CENTERED OPEN TO TRANSNATIONAL AND GLOBAL ALTERNATIVES. European Journal of Open Education and E-learning Studies, [S.1.], dec. 2018. ISSN 2501 9120. Available at: $<$ https://oapub.org/edu/index.php/ejoe/article/view/2137>. Date accessed 14 Apr. 2019.

[13] Irish, Cheryl. and Scrubb, Monica.2012. Five Competencies for Culturally Competent Teaching and Learning. The Teaching Professor. https://www.facultyfoc us.com/

[14] Jimenez, Ray. 2006. 3- Minute e-Learning. https://www.a mazon.com/3-Minute-Learning-Learning-Applications-A mazingly/dp/0979184703 
[15] Jimenez, Ray. 2013. How to Chunk Content into 3 Minutes - Part 2 - Tip \#24

[16] KERR.2017.Top 10 Hard Skills Employers Love-List \& Examples. https://resumegenius.com/how-to-write-a.../skills.../hard-sk ills-list

[17] Kryss Tal. 2018. Inventions. http://www.krysstal.com/displ ay_inventions. php?years

[18] LaMotte, Allison. 2018. Multi-Device E-Learning: What, W https:/community.articulate.com/articles/multi-device-e-le arning-what-why-how

[19] Lynch, Mathew. 2018. 3 WAYS TO BECOME A CULTURALLY RESPONSIVE TEACHER. Edupedia, https://www.theedadvocate.org/edupedia/

[20] McDaniel, Don. 2018. Carl Ransom Rogers. http://www2.southeastern.edu/Academics/Faculty/ /educ692/Rogers.html

[21] Michael, Shearson, and Others. 2011. Barriers to Successful Implementation of Technology Integration in Educational Settings. https://onlinelibrary.wiley.com/doi/abs/10.1111/j cal.12034

[22] Middleton, Andrew. and Glover, Ian. 2017. Teaching in Active Learning Classrooms. https://blogs.shu.ac.uk/learni ngspaces/files/2017/06/ALC-handbook.pdf

[23] Mirza, Abdulrahman A., and Al-Abdulkareem, Mohammed, 2011. Models of e-learning adopted in the Middle East. https://www.researchgate.net/Models_of_e-learning_adopt ed_in_the_Middle_East

[24] NETO, Northeastern Educational Television of Ohio, Inc. 2008. Characteristics of the Feudal World. https://westernreservepublicmedia.org/middleages/feud_fe udalw.htm

[25] Nilson, Linda. 2018. Teaching Critical Thinking: Some Practical Points. Magna Publications. FACULTY FOCUS, Teaching Professor. https://www.facultyfocus.com/articles /effective-teaching-strategies/teaching-critical-thinking-pra ctical-points/?=FF181004

[26] Partridge, Allen and Ramaswamy, Sharath. 2018. 10 Implementation Strategies for Your New Learning program (LMS) https://elearningindustry.com/ /ten-implementation-strategies-for-your-new-learning-

[27] Phillips, Mark. 2014. A Place for Learning: The Physical Environment of Classrooms. https://www.edutopia.org/blo $\mathrm{g} /$ the-physical-environment-of-classrooms-mark-phillips

[28] Rose, Todd. 2016. The End of Average. http://www.thoma sencharles.nl/wp-content/uploads/2017/01/TheEndofAvera geToddRose2016.pdf

[29] SABA.2018. Modernize Your Corporate Learning: The Time is Now. Saba Software Inc. https://www.saba.com/re sources/ebooks- guides/modernizing-corporate-learning.

[30] Samdperil, Gail. and Gunther, Christina. 2017. Intercultural Competencehttps://www.aacu.org/sites/default/files/files/d 1ss17/CS20Presentation.pdf

[31] Stevenson, Beatrix. 2017. Best Practices in Teaching Written and Oral Communication Skills to STEM Students https://www.dreamstime.com/stock-photography-math-equ ations-image.

[32] Smith, M. K. 2004. 'Carl Rogers and informal education', the encyclopedia of informal education. www.infed.org/thinkers/et-rogers.htm.

[33] UNESCO's International Institute for Educational Planning. 2018. Information and communication technology (ICT) in education.https://learningportal.iiep.unesco.org/en/issue-br iefs/improve-learning/curriculum-and-materials/informatio

-and-communication-technology-ict

[34] Vignettes Learning. https://vignettestraining.blogspot.com/ 2013/07/how-to-chunk-content-into-3-minutes_30.html

[35] Wikipedia. 2018. Cultural competence in healthcare. https://en.wikipedia.org/wiki/Cultural_competence_in_hea lthcare

[36] Zook, Chris.2018. 11 principles of classroom management and how you can use them to teach digital natives. https://www.aeseducation.com/blog/author/chris-zook 